\title{
Prevalence of tracheobronchomalacia and excessive dynamic airway collapse in bronchial asthma of different severity
}

\author{
Roberto W Dal Negro ${ }^{1 *}$, Silvia Tognella ${ }^{1}$, Massimo Guerriero ${ }^{2}$ and Claudio Micheletto ${ }^{3}$
}

\begin{abstract}
Background: Tracheobronchomalacia (TBM) is a pathologic condition in which softening of tracheal and bronchial cartilage causes the dynamic narrowing of transverse or sagittal diameters of tracheobronchial lumen; an excessive dynamic airway collapse (EDAC) may also be associated, with a substantial invagination of the posterior membrane of trachebronchial tree.

The aim of this study was to assess the prevalence of both TBM and EDAC in a population of asthmatics with different degrees of disease severity compared to a reference group of subjects without any bronchial obstruction.

Methods: A cohort of 202 asthmatics was investigated by means of a dynamic flexible videobronchoscopy: 74 mild persistent (MPA - age 18-68 ys; 35 males; mean $\mathrm{FEV}_{1}=88.6 \%$ pred. $\pm 8.3 \mathrm{sd}$ ); 63 moderate (MA - age 21-71 ys; 30 males; mean $\mathrm{FEV}_{1}=71.3 \%$ pred. $\left.\pm 9.1 \mathrm{sd}\right), 65$ severe asthmatics $\left(\mathrm{SA}-\right.$ age $33-70 \mathrm{ys} ; 25$ males; mean $\mathrm{FEV}_{1}=48.5 \%$ pred. $\pm 7.6 \mathrm{sd}$ ), and 62 non obstructed subjects (NO - age 18-71 ys; 38 males; mean FEV $198.6 \%$ pred. $\pm 2.7 \mathrm{sd}$ ). TBM and EDAC were classified according to FEMOS classification.
\end{abstract}

Results: TBM and EDAC were observed in only 1/62 subjects (both 1.61\%) of NO group, while their prevalence was $2.70 \%$ and $6.75 \%$ in MPA group; $7.93 \%$ and $19.04 \%$ in MA group; $18.46 \%$ and $69.23 \%$ in SA group, respectively. The crude prevalence of thyroid disorders in the population was 12.9\%. In particular, the prevalence of thyroid disorders was significantly higher in females than in men, but 54-fold higher in females than in men in the presence of EDAC.

Conclusions: 1) The prevalence of both TBM and EDAC is directly related to age, gender (females), and asthma severity; 2) EDAC is much more frequent than TBM in all asthma patients; 3 ) both tracheal abnormalities proved to be more represented in asthmatics with thyroid disorders, and particularly in female asthmatics with EDAC.

Keywords: Bronchial asthma, Collapse, Excessive dynamic airway, Thyroid disorders, Tracheobronchomalacia

\section{Background}

Tracheobronchomalacia (TBM) is a pathologic condition in which softening of tracheal and bronchial cartilage causes the dynamic narrowing of transverse or sagittal diameters of tracheobronchial lumen. This condition may or may not be associated with a substantial invagination of the posterior membrane of trachebronchial tree, an entity referred to an excessive dynamic airway collapse (EDAC), which is peculiar in some patients with

\footnotetext{
* Correspondence: robertodalnegro@gmail.com

${ }^{1}$ Respiratory Unit, Orlandi General Hospital, Via Ospedale 2, Bussolengo VR 37012, Italy

Full list of author information is available at the end of the article
}

obstructive pulmonary disease, such as chronic bronchitis, emphysema, asthma, or can represent an isolated finding in patients during cough or forced expiration.

Tracheomalacia refers to a weakness of the trachea frequently due to a reduction and/or atrophy of the longitudinal elastic fibers of the pars membranacea or impaired cartilage integrity, such that the airway is softer and more susceptible to collapse $[1,2]$. This disease may arise congenitally (from disorders associated with impaired cartilage maturation or in combination with other abnormalities like tracheoesopageal fistula) or it may be acquired from prior intubation, trauma, infections, long standing extrinsic compression, or chronic inflammation

\section{Biomed Central}


$[3,4]$. Localized TBM is usually seen in patients with prolonged endotracheal intubation, tracheostomy, or vascular rings. The cause of diffuse TBM is often unknown, but it is frequently seen in patients with common respiratory conditions, such as chronic bronchitis.

Acquired TBM has been reported to be present in $4.5 \%$ of bronchoscopies in large general series [2]; in $12.7 \%$ of all patients undergoing bronchoscopy evaluation for respiratory disease [3], and in as many as $44 \%$ of patients undergoing bronchoscopy who have a history of chronic bronchitis [4].

It has been shown that chronic airway inflammation with diffuse panbronchiolitis might play a role in exacerbations of this disorder and the acquired form of TBM has been increasingly recognized as an important cause of chronic respiratory symptoms [5]. In TBM, central airway collapse is not closely related to airflow obstruction, and expiratory flow limitation at rest often occurs in peripheral airways without central airway collapse [6].

This pathologic airway weakening can lead to a dynamic outflow obstruction associated with symptoms (such as: dyspnea; orthopnea; intractable cough, and inability to clear secretions) which can sometimes mimic asthma worsening. Furthermore, TBM may also be associated with a substantial invagination of the posterior membrane of the tracheo-bronchial tree, an entity defined as excessive dynamic airway collapse (EDAC). [7]. To our knowledge, the incidence of TBM in patients with a reported history of asthma or Chronic Obstructive Pulmonary Disease (COPD) has not been extensively studied. However, several large studies in the general population suggest that the overall incidence of TBM is $5-10 \%[8,9]$.

However, at present, it is not generally known in which proportion patients with asthma have TBM or EDAC, and if these conditions are related to asthma severity.

The aim of this study was to evaluate the prevalence of both TBM and EDAC in a population of asthmatics of different degrees of severity compared to a reference group of subjects without any airway obstruction.

\section{Methods}

A cohort of 202 non-smoking asthmatics was investigated: 74 mild persistent, MPA (age 18-68 years; 35 males; mean $\mathrm{FEV}_{1}=88.6 \%$ pred. $\left.\pm 8.3 \mathrm{sd}\right) ; 63$ moderate asthmatics, MA (age 21-71 years; 30 males; mean $\mathrm{FEV}_{1}=71.3 \%$ pred. $\pm 9.1 \mathrm{sd}$ ); 65 severe asthmatics, SA (age 33-70 years; 25 males; mean $\mathrm{FEV}_{1}=48.5 \%$ pred. $\pm 7.6 \mathrm{sd}$.

Criteria used for defining asthma in the present study were those accepted worldwide [10]. In particular, asthma diagnosis stemmed from subjects' family and clinical data; from lung function tests (in particular, the variability of their airway obstruction; the reversibility (30' after salbutamol $400 \mathrm{mcg}$ ) of their flow limitation from basal values, and/or the measurement of their bronchial response to methacholine).

All patients were already treated according to GINA guidelines [10]. All patients were in stable conditions at the time of their bronchoscopy.

Another cohort of 62 non smoking subjects without any obstructive disease, NO (age 18-71 years; 38 males; mean $\mathrm{FEV}_{1} 98.6 \%$ pred. $\left.\pm 2.7 \mathrm{sd}\right)$ was also investigated from this point of view (during a bronchoscopy performed as part of an unrelated investigation) as a reference group.

None of the subjects had a systemic disease or localized conditions known as possible cause of TBM. The study was approved by the local E.C. in date January 19th, 2010 (prot. n.: 1489).

\section{Diagnosis}

Bronchoscopic visualization of dynamic tracheal or bronchial collapse remains the gold standard for TBM diagnosis [11]. After the patients had signed the specific informed consent, all bronchoscopies were performed with topical anesthesia (lidocaine) and light sedation (midazolam 1.25 - $2.5 \mathrm{mg}$ ev). The patients were studied in the supine position and had undergone standard spirometry to determine $\mathrm{FEV}_{1}$ the day before broncoscopy. During the examination, patients were able to breathe spontaneously and follow commands to perform deep breathing, forced exhalation, and cough maneuvers to elicit the collapsibility of the airways. The bronchoscope was introduced into the trachea, and at that point the patient was instructed to perform forced inspiratory and expiratory maneuvers with graded degrees of effort, according to a standard TBM protocol [6]. The bronchoscope was then advanced to $5 \mathrm{~cm}$ above the carina, and the patient was requested to perform again graded forced expiratory maneuvers. This procedure was then repeated at the entrance of the right and left main bronchi. All bronchoscopies were video recorded and reviewed after the procedure to assess the degree of airway collapse.

Obviously, the airways collapsed during forced expiration, but in some cases the airway lumen was completely obliterated as the membranous trachea had collapsed to the cartilaginous rings. Dynamic CT scan images can define the normal range of intrathoracic tracheal diameters and cross-sectional areas during forced maneuvers, and it was shown that tracheal narrowing was about $80 \%$ in patients with TBM vs $35 \%$ in healthy men [12]; Stem et al. then recommended using a cutoff of $\geq 70 \%$ narrowing on forced expiration as a diagnostic threshold for TBM [12]. 


\section{Classification}

TBM and EDAC were classified according to FEMOS classification, that addresses Functional status; Extent of abnormalities notes; Morfology of the abnomarlity; Origin of the abnormality, and Severity of the disease process [7].

In the present study, only moderate (expiratory airways collapse $\geq 75 \%$ ) and severe (expiratory airway collapse of $100 \%$, such as, the airway walls make contact) TBM or EDAC were considered.

\section{Statistics}

Quantitative variables were expressed as mean and standard deviation; categorical variables as absolute and relative frequencies. The Skewness test was used to check the normality in distribution for quantitative variables. Data were compared by t-test and Mann-Whitney test, CHI-square test, and Fisher test as appropriate.

The logistic regression model was used to estimate the odds ratio and to control the role of confounding variables; $\mathrm{p}<0,05$ was considered statistically significant. Statistical analysis was carried out by using STATA Release 12 (Stata Corp LP, Texas, USA).

\section{Results}

Bronchoscopic visualization of trachea and main bronchi was possible in all subjects, without significant adverse events. A moderate desaturation and wheezing occurred in 42 subjects immediately after the procedure, and they were quickly recovered with inhaled salbutamol $(400 \mu \mathrm{g}$ via spacer) and prednisone $40 \mathrm{mg}$ e.v. All patients were monitored in the 2 hours following the bronchoscopic examination and were discharged from the Lung Unit only with normal values for systemic blood pressure, heart frequency and peripheral saturation.

General characteristics of non-obstructive and of asthmatic subjects are reported in Table 1. Males were significantly prevailing in subjects of the reference group ( $\mathrm{p}<0.021)$; asthmatics were significantly older $(\mathrm{p}<$ 0.0001 ), and characterized by a lower mean $\mathrm{FEV}_{1}$ value ( $\mathrm{p}<0.0001)$. The overall prevalence of TBM and EDAC proved quite lower (only 1 case) in non asthmatic subjects: therefore, no statistical inference is possible for groups consisting of 1 statistical unit. When considering the whole sample of asthmatics, TBM was present in 19 cases (9.4\%) and EDAC in 62 cases (30.7\%), being one of these abnormalities of central airways detectable in $41.1 \%$ of a general population of asthmatics (Table 1 ).

Moreover, TBM was found in $2(2.70 \%)$ and EDAC in 5 cases $(6.75 \%)$ of mild persistent asthma (MPA), while TBM was found in $5(7.93 \%)$ and EDAC in 12 cases (19.04\%) of moderate persistent asthma (MA). Finally, TBM was found in $12(18.46 \%)$ and EDAC in 45 cases (69.23\%) of severe persistent asthma (SA). Both the TBM and the EDAC prevalence, as well as their ratio, are directly and significantly related (both $\mathrm{p}<0.0001$ ) to asthma severity according to an exponential function, particularly in the case of EDAC (Table 2).

When compared to that of asthmatics without any tracheal abnormalities, the mean age in those with TBM or EDAC was higher $(56.4 \pm 10.2 \mathrm{sd}$ yr vs $44.6 \pm 13.0 \mathrm{sd} \mathrm{yr}$, $\mathrm{p}<0.0001$, and $51.8 \pm 12.5 \mathrm{sd}$ yr vs $43.5 \pm 12.8 \mathrm{sd} \mathrm{yr}$, $\mathrm{p}<0.001$, respectively).

The crude prevalence of thyroid disorders in the population was $12.9 \%(34 / 264)$ : in particular: thyroidectomy $52.9 \%$ (18/34); thyroiditis $26.5 \%$ (9/34); goiter $14.8 \%$ $(5 / 34)$; malignancy $2.9 \%(1 / 34)$; other $2.9 \% \quad(1 / 34)$ (Table 3$)$. When the prevalence of thyroid disorders was investigated by gender, the OR calculated was $=8.77$ (95\% CI $=2.94-35.13$ (by the Fischer Exact Confidence method), such as the probability for the presence of thyroid disturbances was 5-fold higher in females than in males. Moreover, when the prevalence of both EDAC and thyroid disorders was compared by gender, the OR calculated was $=54.76(95 \% \mathrm{CI}=17.48-213.06)$, such as, the probability for the presence of thyroid disturbances was 54-fold higher in females than in males with EDAC.

\section{Discussion}

During normal expiration, there is a physiologic reduction in all dimensions of intrathoracic airways; when changes occurring during forced inspiration and expiration are compared by a dynamic CT, a mean 35\% decrease in the cross-sectional area of the trachea has been described [12]. Thus, a certain degree of dynamic airway collapse, characterized by invagination of the posterior membrane and narrowing of the cross-sectional area of the tracheobronchial tree, represents a physiologic event. However, this phenomenon is exaggerated in some patients suffering from obstructive airway disease, or it represents an isolated finding in some patients during cough and forced expiration.

Table 1 General characteristics of non-obstructive and of asthmatic subjects

\begin{tabular}{|c|c|c|c|c|c|}
\hline & Gender \% m & Age $(y r)$ mean $\pm s d$ & $\mathrm{FEV}_{1} \%$ pred. mean $\pm \mathrm{sd}$ & TBM n (\%) & EDAC n (\%) \\
\hline Non-obstructive $(n=62)$ & 61.3 & $38.9 \pm 10.4$ & $98.6 \pm 3.7$ & $1(1.6 \%)$ & $1(1.6 \%)$ \\
\hline Asthmatics $(n=202)$ & 44.6 & $47.5 \pm 13.3$ & $70.3 \pm 17.7$ & $19(9.4 \%)$ & $62(30.7 \%)$ \\
\hline$p$ & 0.021 & 0.0001 & 0.0001 & - & - \\
\hline
\end{tabular}

General characteristics of asthmatic subjects are reported in Table 2 by asthma severity. Females were significantly more represented in severe asthmatics $(\mathrm{p}<0.01)$. 
Table 2 General characteristics of asthma subjects by their asthma severity, and the corresponding distribution of TBM and EDAC

\begin{tabular}{|c|c|c|c|c|c|}
\hline & Gender $\% \mathrm{~m}$ & Age (yr) mean \pm sd & $\mathrm{FEV}_{1} \%$ pred. mean $\pm \mathrm{sd}$ & TBM n (\%) & EDAC n (\%) \\
\hline Mild Persistent Asthma $(n=74)$ & 47.3 & $40.8 \pm 14.1$ & $88.6 \pm 5.6$ & $2(2.70 \%)$ & $5(6.75 \%)$ \\
\hline Moderate Asthma $(n=63)$ & 47.6 & $49.3 \pm 12.2$ & $71.4 \pm 5.4$ & $5(7.93 \%)$ & $12(19.04 \%)$ \\
\hline Severe Asthma $(n=65)$ & 38.5 & $53.4 \pm 9.9$ & $48.5 \pm 6.8$ & $12(18.46)$ & $45(69.23 \%)$ \\
\hline$p^{*}$ & 0.01 (Mild-moderate vs severe) & 0.0001 & 0.0001 & 0.0001 & 0.0001 \\
\hline
\end{tabular}

*anova.

TBM is an increasingly recognized abnormality of the central airways and generally results from weakness of the tracheal or mainstem bronchial walls caused by either softening of the supporting cartilaginous rings, or redundancy of the connective tissue of the posterior membrane due to a reduction in size and number of elastic fibers, or both. TBM has been called tracheobronchial collapse, expiratory tracheobronchial collapse, expiratory tracheobronchial stenosis, and tracheobronchial dyskinesia. These various terms have not only provided an incomplete understanding of the pathophysiology of malacia and EDAC, but have also contributed to the existing confusion concerning these obstructive airway disorders and to maintain some of the uncertainty on the optimal patients' management. Most published studies are case series and retrospective descriptions; in addition, few investigators regard malacia and EDAC as separate entities, often using the two words interchangeably.

The clinical impact of these abnormalities is quite high. The pathologic weakening of the airway can produce dynamic outflow obstruction and severe symptoms, such as: cough, dyspnea, and wheezing resistant to corticosteroids and inhaled bronchodilators, stridor, recurrent bronchitis or pneumonia, atelectasis, difficult secretions clearing, and respiratory insufficiency. Moreover, air trapping was also observed with a higher frequency and greater severity in patients with tracheobronchomalacia when compared to patients of similar ages without TBM [13], and up to $7 \%$ of patients with severe diffuse TBM may require mechanical ventilation due to respiratory failure. [14]. Current treatments include techniques that splint the central airways, such as continuous positive pressure ventilation [15], silicone airway stents [16] and surgical tracheobronchoplasty [17]. Surgical central airway stabilization with posterior

Table 3 Gender dependency of TBM or EDAC and thyroid disorders

\begin{tabular}{cccccc}
\hline Gender & $\mathbf{n}$ & $\%$ & & $\mathbf{n}$ & $\%$ \\
\hline males & $4 / 128$ & 3.1 & TBM & $4 / 4$ & 100 \\
& & & EDAC & $0 / 4$ & 0.0 \\
females & \multirow{2}{*}{$30 / 136$} & 22.1 & TBM & $7 / 30$ & 23.3 \\
& & & EDAC & $23 / 30$ & 76.7 \\
\hline
\end{tabular}

tracheobronchial splinting using a polypropylene mesh improves respiratory symptoms, health-related quality of life, and functional status in highly selected patients with severe symptomatic TBM [18].

The etiology of acquired TBM in many patients is not obvious. TBM is often seen in association with COPD in patients with a smoking history, thus leading to the inference that chronic inflammation and smoking are important contributing factors [19].

Nevertheless, little is known concerning the mechanism underlying the association between inflammation and TBM or EDAC. Loring et al. [6] speculated that repeated mechanical stress from coughing or the high expiratory pleural pressures during exercise in patients with airflow obstruction might cause stretching and degeneration of the posterior membranous portion of the trachea and main bronchi over a period of years.

At present very few data concerning TBM or EDAC epidemiology in asthma patients are available to our knowledge. Data from the present study, carried out in a consistent cohort of asthma patients of different severity and in whom the diagnosis of TBM o EDAC was confirmed via dynamic broncoscopy, ascertained that the prevalence of EDAC is directly related to asthma severity and is particularly high in severe asthma, while EDAC is much more frequent than TBM in asthmatic patients independently of asthma severity. This evidence supports the hypothesis that the chronic persistency of substantial airway inflammation within the airways can contribute to impair expiratory airflow just affecting intrinsically and persistently the structures of larger airways in a great proportion of cases.

Thyroid disease and its association with TBM was documented, but only when tracheal cartilaginous ring can be compromised by a tumour, or most commonly after thyroidectomy, especially in patients with cancer [19]. Furthermore, in our study, thyroid disorders proved highly concomitant in TBM and in EDAC, the latter condition being much more represented in females, independently of asthma severity.

\section{Conclusions}

In conclusion, the presence of TBM or EDAC should be considered whenever bronchial asthma persists 
uncontrolled and unresponsive to an appropriate pharmacological treatment. Moreover, in severe asthma the presence of TBM or EDAC should be actively investigated, not only when uncontrolled, bearing in mind the range of non pharmacological treatment options that could be offered to these patients. Furthermore, if the occurrence of EDAC is highly probable in females with thyroid disturbances, it is as much appropriate that thyroid function be investigated in females with EDAC.

\section{Competing interests}

The authors declare that they have no competing interests.

\section{Author details}

${ }^{1}$ Respiratory Unit, Orlandi General Hospital, Via Ospedale 2, Bussolengo VR 37012, Italy. 'Economics Department, Statistical Section, University of Verona, Italy. ${ }^{3}$ Respiratory Unit, Mater Salutis Hospital, Legnago, Verona, Italy.

Received: 2 January 2013 Accepted: 18 March 2013

Published: 14 May 2013

\section{References}

1. Johnson TH, Mikita JJ, Wilson RJ, Feist JH: Acquired tracheomalacia. Radiology 1973, 109:576-580.

2. Jokinen K, Palva T, Sustinen S, Nuutinen J: Acquired tracheobronchomalacia. Ann Clin Res 1977, 9:52-57.

3. Ikeda S, Inuue Y, Fujino S, Fujioka S, Hamada H, Yokoyama A, Kohno N Hiwada K: A case of diffuse panbronchiolitis associated with tracheomalacia. Nihon Ronen Igakkai Zasshi 1993, 30:974-977.

4. Jokinen K, Palva T, Nuutinen J: Chronic bronchitis. Abronchologic evaluation. J. Otorhinolaryngol Relat Spec 1976, 38:178-186.

5. Palombini BC, Villanova CA, Araùjo E, Gastal OL, Alt DC, Stolz DP, Palombin CO: A pathogenic triad in chronic cough: asthma, postnasal drip and gastroesophageal reflux disease. Chest 1999, 116:279-284.

6. Loring SH, O'Donnel CR, Feller-Kopman DJ, Ernst A: Central airway mechanics and flow limitation in acquired tracheobronchomalacia. Chest 2007, 131:1118-1124.

7. Murgu DS, Colt GH: Tracheobronchomalacia and excessive dynamic airway collapse: novel diagnostic tools clarify the issues. Pulm Perspectives 2005, 22,3:7-10.

8. Hasegawa I, Boiselle P, Raptopoulos V, Hatabu H: Tracheomalacia incidentally detected on $C T$ pulmonary angiography of patients with suspected pulmonary embolism. AJR Am J Roentgenol 2003, 181:1505-1509.

9. Ikeda SH, Hanawa T, Konishi T, Adachi M, Sawai S, Chiba W, Kosaba S, Hatakenaka R, Matsubara Y, Funatsu T: Diagnosis, incidence, clinicopathology and surgical treatment of acquired tracheobronchomalacia. Nihon Kyobu Shikkan Gakka iZasshi 1992 30:1028-1035.

10. Global Strategy for asthma management and prevention: Global Initiative for Asthma (GINA): NHLB/WHO Workshop Report. National Heart, Lung, and Blood Institute, National Institutes of Health, Updated 2010. Available from www.ginasthma.org.

11. Carden KA, Boiselle PM, Waltz DA, Ernst A: Tracheomalacia and tracheobronchomalacia in children and adults: an in-depth review. Chest 2005, 127:984-1005.

12. Stern EJ, Graham CM, Webb WR, Gamsu G: Normal trachea during forced expiration: dynamic CT measurements. Radiology 1993, 187:27-31.

13. Zhang J, Hasegawa I, Hatabu H, Feller-Kopman D, Boiselle PM: Frequency and severity of air trapping at dynamic expiratory $\mathrm{CT}$ in patients with tracheobronchomalacia. AJR Am. J Roentgenol 2004, 182:81-85.

14. Ernst A, Majid A, Feller-Kopman D, Guerrero J, Boiselle P, Loring SH, O'Donnell C, Decamp M, Herth FJ, Gangadharan S, Ashiku S: Airway stabilization with silicone stents for treating adult tracheobronchomalacia: a prospective observational study. Chest 2007, 132:609-616.

15. Fergusson GT, Benoist J: Nasal continuous positive airway pressure in the treatment of tracheobronchomalacia. Am Rev Respir Dis 1993. 147:457-461.
16. Sommer D, Forte $\mathrm{V}$ : Advances in the management of major airway collapse: the use of airway stents. Otolaryngol Clin. North Am 2000, 33:163-177.

17. Wright CD: Tracheomalacia. Chest Surg Clin North Am 2003, 13:349-357.

18. Majid A, Guerrrero J, Gangadharan S, Feller-Kopman D, Boiselle P, DeCamp M, Ashiku S, Michaud G, Herth F, Ernst A: Tracheobronchoplasty for severe tracheobronchomalacia. Chest 2008, 134:801-807.

19. McHenry CR, Piotrowski JJ: Thyroidectomy in patients with marked thyroid enlargement: airway management, morbidity, and outcome. Am Surg 1994, 60:586-591.

doi:10.1186/2049-6958-8-32

Cite this article as: Dal Negro et al:: Prevalence of tracheobronchomalacia and excessive dynamic airway collapse in bronchial asthma of different severity. Multidisciplinary Respiratory Medicine 2013 8:32.

\section{Submit your next manuscript to BioMed Central and take full advantage of:}

- Convenient online submission

- Thorough peer review

- No space constraints or color figure charges

- Immediate publication on acceptance

- Inclusion in PubMed, CAS, Scopus and Google Scholar

- Research which is freely available for redistribution 